\title{
P22
}

\section{Gas Injection Laboratory Experiments on Opalinus Clay - Experimental Set-up and Preliminary Results}

\author{
E. Romero* (UPC Barcelona Tech), E.E. Alonso (UPC Barcelona Tech) \& \\ P. Marschall (NAGRA Science and Technology)
}

\section{SUMMARY}

Preliminary innovative tests are currently being performed on undisturbed Opalinus clay. These experiments intend to determine the conditions under which gas breakthrough processes occur, as well as to determine the relevant hydro-mechanical parameters that appear to affect these processes. An instrumented high-pressure triaxial cell is used, which was specifically designed to apply isotropic / anisotropic stress states while injecting gas at controlled volume rate. The study is mainly focused on maximum peak pressures, time for breakthrough, measured flows into and out of the specimen, preferential pathways created, induced desaturation of the sample, and relationship of initial peak pressure with total stress state. 
The multiple barrier concept is the cornerstone of the different proposed schemes for underground disposal of radioactive wastes. One of these barriers is the geological one -the low-flow host formation-, which is the final impediment to radionuclide migration. However, within the context of gas generation and migration, this particular low permeability concept becomes somewhat problematic. If the gas production rate exceeds the rate of diffusion of gas in the host rock, gas would continue to accumulate and pressure increase until it becomes sufficiently large to create pathways. This gas breakthrough pressure appears to depend on the gas injection rate, the stress state, the orientation of rock discontinuities, the degree of saturation, the porosity, and so on. Understanding migration is thus one of the key issues in the assessment of repository performance and is the focus of this research.

Preliminary innovative tests are currently being performed on undisturbed Opalinus clay. These experiments, besides providing quantitative data to be used in the validation of numerical models for the simulation of gas migration (see for example Arnedo et al. 2008), they intend to determine the conditions under which gas breakthrough processes occur, as well as to determine the relevant hydro-mechanical parameters that appear to affect these processes (e.g. water retention curves, intrinsic and relative permeability, mechanical parameters). The gas injection study performed using controlled volume rates is mainly focused on maximum peak pressures, time for breakthrough, measured flows into and out of the specimen, aperture and preferential pathways created, induced desaturation of the sample, effects of a second injection (depressed injection peak due to partially open pathways), relationship of initial peak pressure with total stress state (the gas pressure has to reach the total stress in a critical plane in order to develop a preferential path), and so on. For example, desaturation is a critical issue, since previous tests performed by Horseman et al. (1999) and Harrington and Horseman (2003) evidenced that no water displacement occurred inside the specimen, despite the fact that the observed breakthrough pressure appeared to be higher than the air entry pressure of the material. In addition, Graham et al. (2002) found that the breakthrough pressure decreased as the degree of saturation decreased. It is expected that for high degrees of saturation, gas migration takes place as a combination of two-phase flow and some preferential paths created by the gas pressure.

An instrumented high-pressure triaxial cell is being used, which is specifically designed to apply isotropic / anisotropic stress states while injecting gas at controlled volume rate. Axial and radial stresses are applied with pneumatic / hydraulic systems up to a maximum of 20 MPa. The axial deformation of low-height specimen is registered with an external LVDT. Each cap of the cell has inlet and outlet lines, prepared for gas or liquid connections. The equipment uses four automatic pressure / volume controllers, two for gas (injection and extraction at downstream point) and two for water (injection and recovery at downstream point). For sample saturation and water permeability determination two low water pressure / volume controllers are connected to the bottom (water inflow system) and top (water outflow) caps, respectively. The high gas pressure / volume controller is connected to the bottom cap during the air injection tests, while a low pressure / volume controller is used to measure the gas or liquid flow at the downstream point. The system is complemented with an air / water interface that is used to measure the water outflow during the gas injection tests (the recovery system is initially full of water, and the excess water mass is measured by an electronic balance). In this way, the saturation state is monitored during gas injection. Gas recovery is performed at constant and low gas pressure. The gas injection pressure / volume controller has a maximum range of $20 \mathrm{MPa}$, and is able to control volume rates between $10^{-4} \mathrm{~mL} / \mathrm{min}$ and $100 \mathrm{~mL} / \mathrm{min}$ (volume resolution $<5 \mathrm{~mm}^{3}$ ). Figure 1 shows a picture of the newly developed triaxial cell jointly with the test set-up.

Opalinus clay samples obtained by single coring technique with air (diameter $150 \mathrm{~mm}$ ), cast in resin and oriented normal to bedding were carefully prepared to fit the required cylindrical dimensions (50 $\mathrm{mm}$ in diameter and maximum height $25 \mathrm{~mm}$ ) with minimum perturbations to avoid affecting their integrity. Samples were pre-cut under dry conditions with a band saw, 


\section{Fault and Top Seals \\ Montpellier 2009}

then prepared with a lathe, and then stored with plastic under vacuum. The initial porosity was around 0.17, water content between 6.6 and $6.9 \%$ and total suction around $60 \mathrm{MPa}$. Saturation of the sample is ensured by applying a controlled water gradient between bottom and top ends of the sample, and by measuring equivalent inflow and outflow liquid volumes under stationary flow conditions. The outlet (top cap) is maintained under nearly atmospheric conditions. Increasing water pressures are applied at the bottom cap (max. water pressure $2 \mathrm{MPa}$ ). All this information is used to determine the hydraulic properties of the material.

Different stress states (isotropic and anisotropic stress states with a maximum stress of $20 \mathrm{MPa}$ ) and different bedding orientations (flow parallel or normal to bedding) are envisaged. Protocols for the gas injection tests are continuously updated once some knowledge regarding the test configuration is gained (gas injection volume rates, confining pressures, and orientation of the samples).

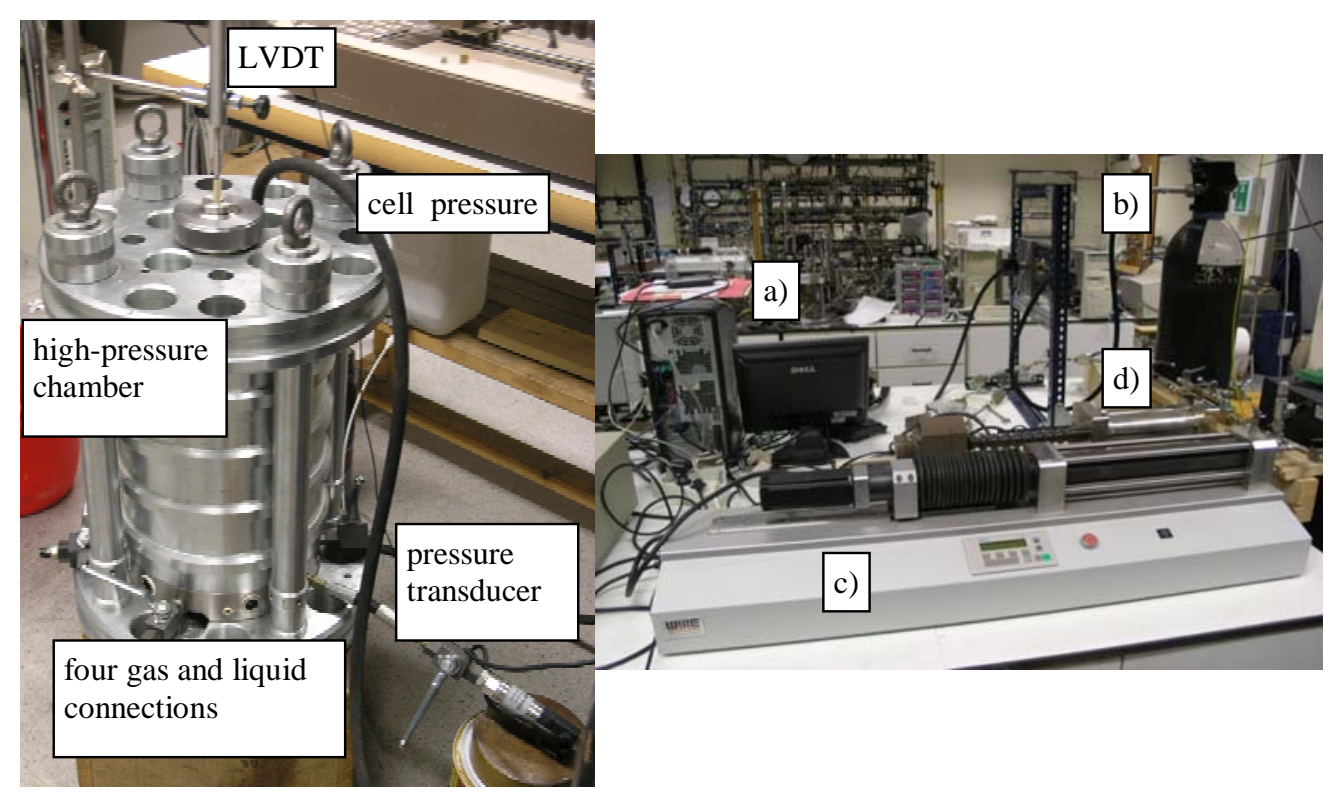

Figure 1. Triaxial cell (at the left) and test set-up: a) data acquisition system; b) pneumatic system with pressure regulators (confining and axial stress); c) high gas pressure / volume controller VPC 500/200 Wille Geotechnik; d) low gas pressure / volume controller GDS Instruments.

Arnedo, D., Alonso, E.E., Olivella, S. and Romero, E. (2008). Gas injection tests on sand/bentonite mixtures in the laboratory. Experimental results and numerical modelling. Physics and Chemistry of the Earth, Parts A/B/C, 33 (Supplement 1): S237-S247.

Graham, J., Gray, M., Halayko, K.G., Hume, H., Kirkham, T. and Oscarson, D. (2002). Gas breakthrough pressures in compacted Illite and Bentonite. Engineering Geology, 64: 273286.

Harrington, J.F. and Horseman, S.T. (2003). Gas migration in KBS-3 buffer bentonite. Sensitivity of test parameters to experimental boundary conditions. SKB Technical Report TR-03-02, Stockholm, Sweden.

Horseman, S.T., Harrington, J.F. and Sellin, P. (1999). Gas migration in clay barriers. Engineering Geology, 54: 139-149. 